\title{
SISTEMA FUZZY PARA ESTIMATIVA DO BEM-ESTAR DE MATRIZES PESADAS
}

\author{
DANILO F. PEREIRA ${ }^{1}$, CAMILA A. BIGHI ${ }^{2}$, LUÍS R. GABRIEL FILHO ${ }^{3}$, \\ CAMILA P. C. GABRIEL ${ }^{4}$
}

\begin{abstract}
RESUMO: Entender o comportamento e suas pequenas variações decorrentes das mudanças do ambiente térmico e desenvolver modelos que simulem o bem-estar a partir de respostas das aves ao ambiente constituem o primeiro passo para a criação de um sistema de monitoramento digital de aves em galpões de produção. Neste trabalho, foi desenvolvido um sistema de suporte à decisão com base na teoria dos conjuntos fuzzy para a estimativa do bem-estar de matrizes pesadas em função de frequências e duração dos comportamentos expressos pelas aves. O desenvolvimento do sistema passou por cinco etapas distintas: 1) organização dos dados experimentais; 2) apresentação dos vídeos em entrevista com "especialista"; 3) criação das funções de pertinência com base nas entrevistas e na revisão da literatura; 4) simulação de frequências de ocorrências e tempos médios de expressão dos comportamentos classificados como indicadores de bem-estar utilizando equações de regressão obtidas na literatura, e 5) construção das regras, simulação e validação do sistema. O sistema fuzzy desenvolvido estimou satisfatoriamente o bem-estar de matrizes pesadas, tendo na sua última versão, com maior número de regras, acertado 77,8\% dos dados experimentais, comparados com as respostas esperadas por um especialista. O sistema pode ser utilizado como instrumento matemático-computacional para apoiar decisões em galpões de produção de matrizes pesadas.
\end{abstract}

PALAVRAS-CHAVE: lógica fuzzy, bem-estar animal, simulação, termoneutralidade, avicultura.

\section{SYSTEM FUZZY FOR ESTIMATE OF WELFARE OF BROILER BREEDERS}

\begin{abstract}
To understand the behavior and its small variations originated from changes of the thermal environment and to develop models that simulate the welfare from broiler breeders' answer to the environment constitute the first step for the creation of a system for digital monitoring of broiler breeders housing. In this paper it was developed a system of decision support based on the fuzzy conjuncts' theory to estimate the welfare of broiler breeders in function of frequencies and times of occurrences of behaviors. The controller's development passed for five distinct steps: 1) organization of the experimental data; 2) observation of the videos in interview with specialist; 3 ) creation of the relevancy functions according to the interviews and the literature revision; 4) frequency simulation of occurrences and average times of expression of the classified behaviors such as indicators of welfare using regression equation from the literature, and 5) construction of rules, simulation and validation of the controller step by step, comparing the results between the simulations. The developed controller satisfactorily estimated the welfare of broiler breeders, having in its version with larger number of rules, estimated about $80 \%$ of the experimental data correctly when compared with the answers expected by a specialist. The system can be used as mathematician-computational instrument to support decisions in broiler breeders housing.
\end{abstract}

KEYWORDS: fuzzy logic, animal welfare, models of simulation, thermoneutral zone, poultry production.

\footnotetext{
${ }^{1}$ Eng ${ }^{\circ}$ Agrícola, Prof. Doutor, UNESP, Câmpus Experimental de Tupã - SP, Fone: (0XX14) 3404.4200, Ramal 4218, danilo@tupa.unesp.br

${ }^{2}$ Graduanda em Administração, Faculdade de Ciências Agrárias e Veterinárias, UNESP, Jaboticabal - SP.

${ }^{3}$ Licenciado em Matemática, Prof. Substituto, Campus Experimental de Tupã, UNESP, Tupã - SP.

${ }^{4}$ Licenciada em Matemática, Profa. Substituta. Campus Experimental de Tupã, UNESP, Tupã - SP.

Recebido pelo Conselho Editorial em: 19-11-2007

Aprovado pelo Conselho Editorial em: 8-12-2008
}

Eng. Agríc., Jaboticabal, v.28, n.4, p.624-634, out./dez. 2008 


\section{INTRODUÇÃO}

Os limites de termoneutralidade são fixados em medidas indiretas, como a temperatura do ar, ou índices de conforto que correlacionam duas ou mais variáveis ambientais. Isso acarreta a imprecisão do provimento das reais necessidades ambientais para as aves, ocorrendo, portanto, o desperdício de energia, seja ela na forma de energia metabólica contida na ração fornecida para os animais e gasta para a manutenção da temperatura corporal, seja na forma de energia elétrica que é utilizada no funcionamento dos sistemas de climatização que são acionados indevidamente. Outro ponto importante a considerar é que todos os animais homeotermos possuem capacidade de se adaptar ao ambiente (CAMPOS, 2000). Assim, os limites de termoneutralidade para uma ave alojada na região Centro-Oeste do Brasil são completamente diferentes dos limites de uma ave alojada na região Sul, mesmo sendo da mesma linhagem, devido a diferenças observadas entre os climas das duas regiões. PEREIRA \& NÄÄS (2008) observaram o deslocamento do limite superior da zona de termoneutralidade de matrizes pesadas quando essas foram submetidas a altas temperaturas por longo período de tempo. Isso ocorre devido à capacidade de aclimatação que os homeotermos possuem, conforme relataram TEETER \& BELAY (1993) e MACARI et al. (1994).

Para aumentar a precisão na estimativa do estresse em aves criadas em aviários, a observação do comportamento das aves tem sido utilizada por vários granjeiros (CAMPOS, 2000). Segundo GRAVES (1982), o comportamento é uma janela entre o organismo vivo e o exterior, e pode ser utilizado para interpretar estados de estresse de animal (DUNCAN \& MENCH, 1993). O comportamento animal era definido até algum tempo como sendo apenas os movimentos executados por um organismo vivo. Porém, uma série de manifestações que podem ser interpretadas como sinais na forma de sons e ruídos, mudanças de cor, odores e produção, que são meios de expressões dos animais e que não são caracterizadas por movimentos, foram incorporados na definição de comportamento (COSTA, 2003).

Entender o comportamento e suas pequenas variações decorrentes das mudanças do ambiente térmico e desenvolver modelos que simulem o bem-estar a partir de respostas das aves ao ambiente, constituem o primeiro passo para a criação de um sistema de monitoramento digital de aves em galpões de produção que identifiquem diferentes níveis de bem-estar das mesmas.

Alguns autores descreveram sistemas de apoio à decisão para sistemas de produção animal, porém poucos são destinados a estimar a sensação de bem-estar. BROWN-BRANDL et al. (2005) testaram cinco modelos de simulação do bem-estar de 128 bezerras, sendo dois modelos de regressão estatística, dois modelos de inferência fuzzy e um modelo de rede neural. Os autores definiram como parâmetro de bem-estar a frequência respiratória, que foi medida durante o experimento. Os modelos foram construídos com $70 \%$ dos dados coletados e testados com 30\%. Desses modelos, o de inferência fuzzy, sem dependência dos dados (mamdani), apresentou $27 \%$ de variação, a menor dentre os modelos testados. AERTS et al. (2003) descreveram a modelagem de uma base de dados para o gerenciamento integrado da produção de calor e do crescimento de frangos de corte. O modelo de controle do crescimento, baseado em resultados de 30 experimentos, foi testado em experimentos realizados em câmara climática. Os resultados mostraram correlações $\mathrm{R}^{2}$ acima de $86 \%$.

A utilização de dados intrínsecos aos animais parece ser o ponto fundamental para conseguir mensurar a sensação de bem-estar (BRACKE et al., 2004; PEREIRA et al., 2005). Utilizando sensores para o monitoramento com precisão da frequência de algum comportamento, pode-se inferir que o aumento ou a diminuição dessa frequência implica aumento da sensação de bem-estar. PEREIRA (2005) estudou as variações de expressão dos comportamentos de matrizes pesadas da linhagem Hybro-PG em função do ambiente físico e determinou cinco comportamentos que são mais afetados pelo ambiente, quais sejam: frequências de ocorrências de deitar, ciscar e uso do ninho e duração das ocorrências de limpar penas e uso do bebedouro. Para esses comportamentos, o autor construiu modelos de regressão linear que simulam as ocorrências em função do ambiente físico. 
$\mathrm{Na}$ busca de contribuir com o tema, este trabalho apresenta um sistema de suporte à decisão com base na teoria dos conjuntos fuzzy para a estimativa do bem-estar de matrizes pesadas em função de frequências e duração dos comportamentos expressos pelas aves.

\section{MATERIAL E MÉTODOS}

\section{Experimentação}

Para a construção do sistema de estimativa do bem-estar das matrizes pesadas, foram utilizados os dados registrados por PEREIRA (2005), obtidos em experimento conduzido em julho de 2005, durante nove dias, em uma câmara climática situada na FEAGRI-UNICAMP, localizada na latitude $22^{\circ} 53^{\prime} \mathrm{S}$, longitude $47^{\circ} 03^{\prime}$ 'W e altitude de $698 \mathrm{~m}$.

No interior da câmara climática, com o objetivo de dividir o local para promover a mesma condição ambiental para três grupos de aves, foram construídos três boxes e três ninhos em estruturas de madeira (Figura 1). Essas estruturas foram cercadas com tela de arame liso hexagonal para pintinho. As dimensões dos boxes eram 1,32 m de largura, 2,14 m de comprimento e 1,5 m de altura.

Em cada box, foram alojadas dez matrizes pesadas e dois galos, o que proporcionou densidade de 5 aves $\mathrm{m}^{-2}$ e 5 matrizes por galo, aproximando-se das densidades observadas nas granjas. $\mathrm{O}$ experimento foi conduzido com três linhagens diferentes, Cobb-500, Hybro-PG e Ross, com idades de 26; 29 e 32 semanas, respectivamente, alojando-se cada linhagem em um box. Porém, para a construção do sistema de suporte à decisão com base na teoria dos conjuntos, foram utilizados apenas os dados referentes à linhagem Hybro-PG, pois as diferentes linhagens respondem de diferentes maneiras aos estímulos do ambiente (KEER-KEER et al., 1996; McGARY et al., 2003). O fotoperíodo simulado foi de $14 \mathrm{~h}$ de luz e $10 \mathrm{~h}$ de escuro, sendo mantido o arraçoamento da granja. Na simulação do ambiente, foram aplicadas três condições de temperatura na câmara climática, ou seja: $26,3{ }^{\circ} \mathrm{C} ; 29,5^{\circ} \mathrm{C}$ e $33{ }^{\circ} \mathrm{C}$ com erros de $\pm 1{ }^{\circ} \mathrm{C}$, com a umidade relativa fixada em $75 \% \pm 5 \%$. Cada condição ambiental foi mantida por três dias.

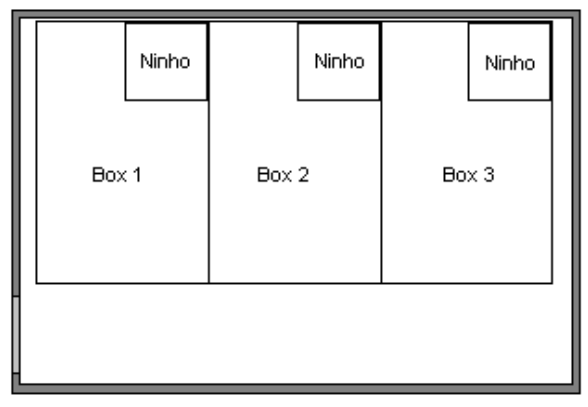

A

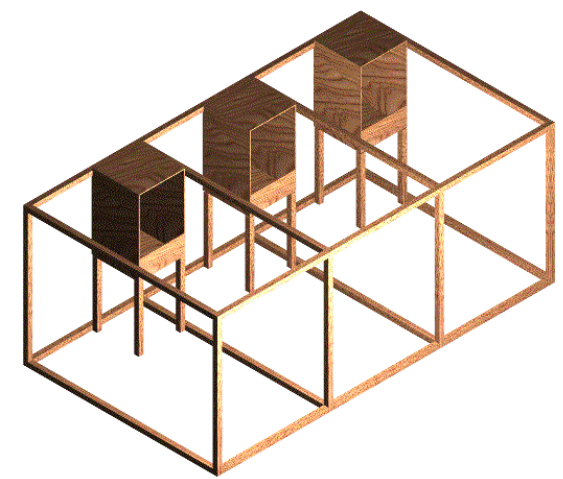

B

FIGURA 1. Disposição dos boxes no interior da câmara climática (PEREIRA, 2005). Layout of boxes in the interior of the climatic chamber (PEREIRA, 2005).

Para o monitoramento dos comportamentos das matrizes, foram instaladas, no teto da câmara climática, três câmeras de vídeo, padrão RGB, da fabricante Mythos ${ }^{\circledR}$, com resolução de 420 linhas, lente convergente de 3,6 mm e sensibilidade mínima de 1 lux, uma sobre cada box. As aves foram monitoradas por essas câmeras durante todo o período de luz, e os vídeos foram gravados em computador.

A variação da temperatura de bulbo seco e da umidade relativa foi monitorada por meio de termoigrômetros modelo Hobo Pro, da fabricante Onset ${ }^{\circledR}$, instalados no centro de cada um dos boxes, permitindo a verificação da homogeneidade do ambiente no interior da câmara climática. A concentração de amônia também foi monitorada dentro da câmara climática e verificou-se a 
influência desse gás nos comportamentos. Essa medida foi feita utilizando bomba de acuro, modelo 31, da fabricante Dräger Röhrchen ${ }^{\circledR}$, com capacidade de sugar $100 \mathrm{~mL}$ de ar, e tubos reagentes da mesma fabricante.

Os vídeos foram gravados e gerenciados pelo software Vigia Digital ${ }^{\circledR}$, que foi fornecido juntamente com as câmeras de vídeo, como parte do pacote para circuito fechado de TV (CFTV). Desses vídeos gravados, foram selecionados trechos de 15 minutos no período da manhã e 15 minutos no período da tarde, em todos os dias do experimento, uma vez que as aves, devido à restrição alimentar realizada na granja e que foi reproduzida no experimento, alimentavam-se apenas no período da manhã, e seus comportamentos diferenciavam-se no decorrer do dia. Esses vídeos foram assistidos utilizando-se de software desenvolvido para o gerenciamento de dados experimentais, denominado Pro-Experimento, e as frequências de ocorrências e tempos de expressão de todos os comportamentos observados foram registrados em banco de dados. Todos os demais registros zootécnicos e do ambiente também foram registrados e gerenciados por esse software, que armazenou todos os dados experimentais em Banco de Dados relacional FireBird ${ }^{\circledR}$.

\section{Construção do sistema de suporte à decisão}

A lógica fuzzy, também denominada difusa ou nebulosa, permite trabalhar com ambiguidade, ou seja, possibilita a perspectiva de estrutura quantitativa alternativa que substitui a lógica aristotélica do meio excludente (é ou não é) pela lógica de Bertrand Russel, citado por LANZILLOTTI \& LANZILLOTTI (1999), na qual as afirmações vagas podem ter valores pertinentes ao intervalo entre zero e um. Nesse cenário, os extremos designam a ausência, e a plenitude da pertinência, respectivamente.

Cinco comportamentos, descritos por PEREIRA (2005) como indicadores do bem-estar das matrizes pesadas, foram utilizados como variáveis de entrada do sistema de estimativa do bem-estar. Além dos comportamentos, as variáveis ambientais temperatura e concentração de amônia também foram utilizadas como variáveis independentes de entrada do sistema.

Todas as variáveis de entrada são definidas no sistema fuzzy por funções de pertinência, nas quais conjuntos de possibilidades categóricas de cada variável foram definidos por funções triangulares (variáveis comportamentais) ou trapezoidais (variáveis ambientais). Para universalizar as funções de pertinência das variáveis comportamentais, os domínios foram recalculados por meio de equações de isomorfismo, para variarem no intervalo [0,1]. Esse artifício matemático fez com que as frequências e as durações dos cinco comportamentos indicadores do bem-estar variassem entre os valores 0 e 1 .

Neste trabalho, a construção das regras do sistema fuzzy, denominado sistema fuzzy sem dependência dos dados (mamdani), seguiu três etapas conforme descreve a Figura 2. As primeira e segunda etapas correspondem à metodologia tradicional de construção de modelos fuzzy (AMENDOLA et al., 2004; AMENDOLA \& SOUZA, 2004). Nessa metodologia, são aproveitados dados de vários experimentos para a elaboração das funções de pertinência e das regras do sistema fuzzy e, na maioria dos casos, são realizadas entrevistas com especialistas. Portanto, essa metodologia é baseada na observação das variáveis e na experiência subjetiva dos especialistas. Na primeira etapa, foram construídas 25 regras, e na segunda, 10 regras.

A terceira etapa descrita na Figura 2 corresponde à inovação metodológica proposta por este trabalho, que visa a aumentar a quantidade de regras do sistema fuzzy, simulando as frequências e os tempos médios de expressão dos comportamentos utilizando os modelos estatísticos de regressão propostos por PEREIRA (2005).

A hipótese inicial é que, a partir dos dados simulados por equações de regressão, no domínio das variáveis, tem-se a possibilidade de aumentar a eficiência do sistema fuzzy com o aumento direcionado das regras. Cada combinação diferente de condição ambiental e comportamental fuzzificados corresponde a uma nova regra para o sistema. Com essa metodologia, foram construídas 82 regras, que foram apresentadas para um especialista que relatou a sua opinião sobre 
o bem-estar de acordo com as condições ambientais e comportamentais combinadas nas regras. Porém, após as simulações dos comportamentos e a entrevista realizada na terceira etapa, constatou-se que as funções de pertinência do comportamento ciscar foram mal calculadas; após sua correção, refizeram-se as simulações com os modelos estatísticos e 52 regras diferentes foram geradas. Essas novas regras foram apresentadas para um especialista que relatou seu parecer do bem-estar sobre as condições combinadas.

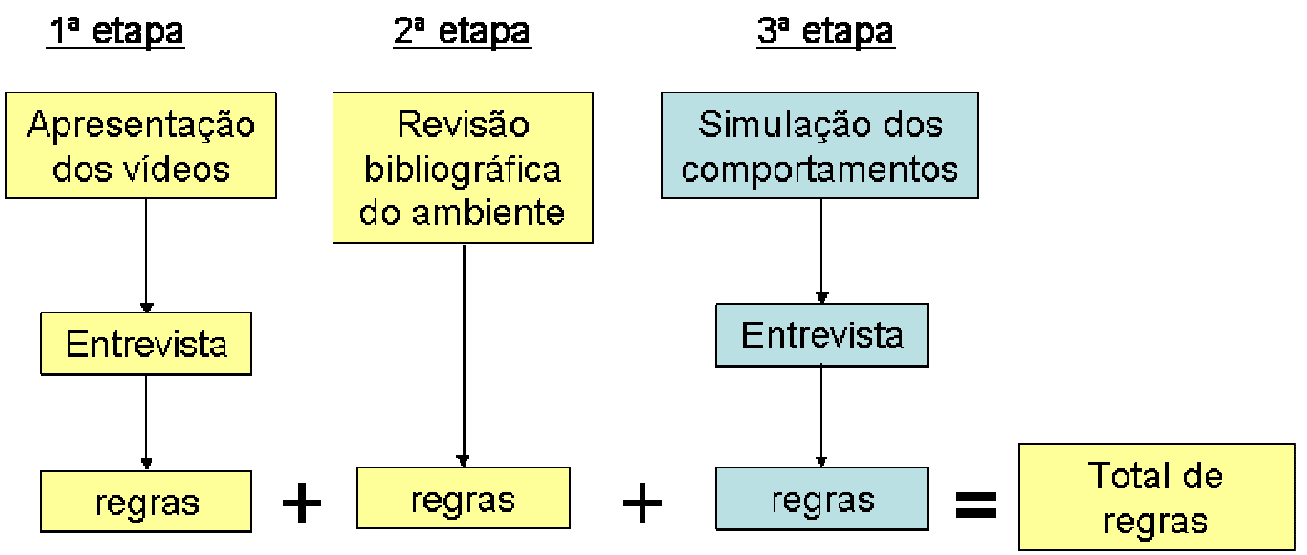

FIGURA 2. Etapas da construção dos sistemas fuzzy. Stages of the construction of the fuzzy system.

Este trabalho testou a eficiência do sistema fuzzy em cada fase da sua construção e, aproveitando-se do equívoco no cálculo da função de pertinência, as 82 regras, que representam situações com baixa possibilidade de ocorrer devido o comportamento ciscar não representar a realidade dos dados, foram utilizadas em duas simulações para testar a hipótese proposta. No total, cinco simulações foram realizadas: uma simulação para as regras da primeira etapa, uma simulação para as regras combinadas da primeira e da segunda etapas e três simulações com as regras combinadas da segunda simulação, sendo uma com as 82 regras obtidas na primeira entrevista da terceira etapa, outra com as 52 regras obtidas na segunda entrevista da terceira etapa e a última simulação combinando todas as regras obtidas.

As simulações foram realizadas inserindo-se no sistema os valores experimentais, sendo os resultados comparados com as respostas dadas pelos especialistas. Para cada simulação, o índice de acertos foi calculado. Na Figura 3, mostra-se a função de pertinência da saída do sistema fuzzy que representa a estimativa do bem-estar das matrizes pesadas da linhagem Hybro-PG.

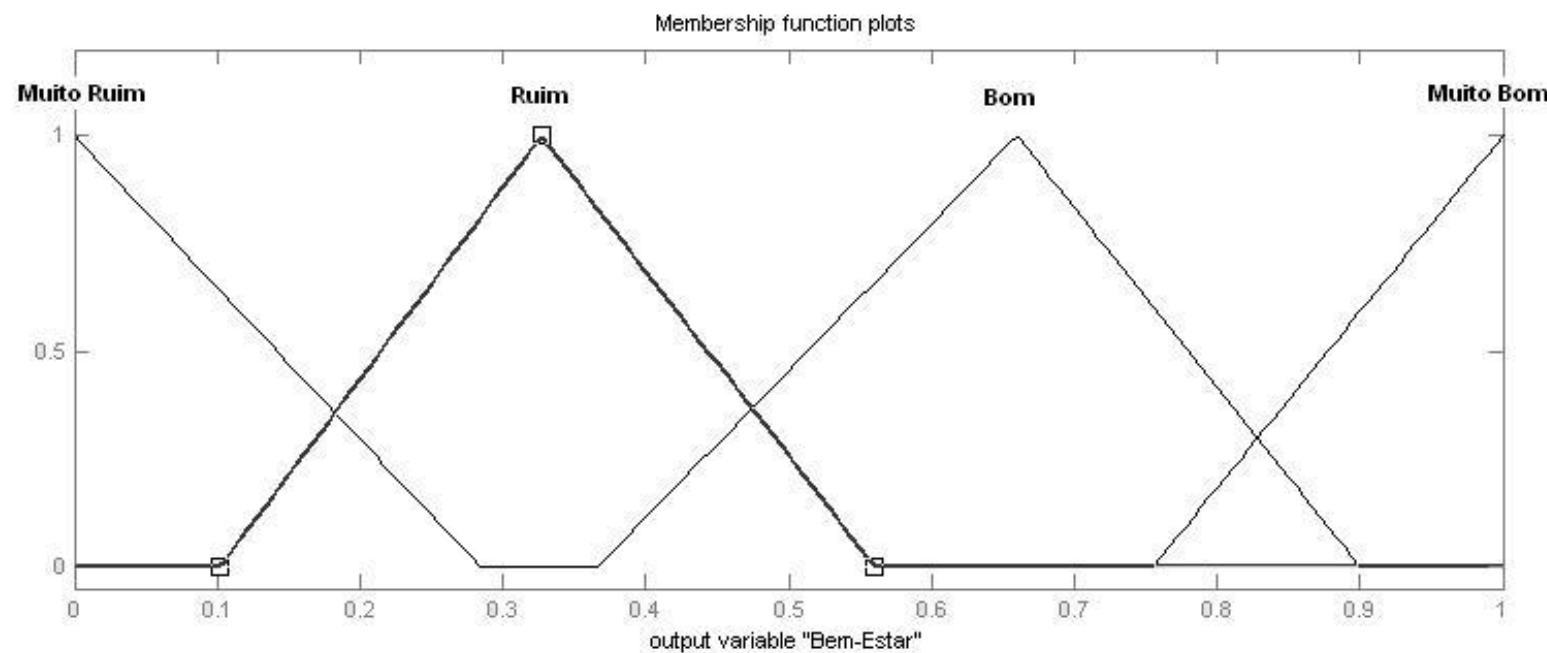

FIGURA 3. Função de pertinência da variável dependente linguística bem-estar. Function of relevancy of the dependent variable linguistic welfare. 
A estimativa de bem-estar (saída do sistema fuzzy) foi definida no domínio [0,1], sendo 1 a condição de bem-estar máximo. Para a variável dependente bem-estar, foram utilizados quatro conjuntos fuzzy, de acordo com as possibilidades de resposta oferecidas nas entrevistas (Figura 3).

\section{RESULTADOS E DISCUSSÃO}

Para testar o sistema fuzzy, todos os dados experimentais (TABELA 1) foram utilizados para as simulações, e as saídas foram confrontadas com as respostas linguísticas dadas pelos especialistas nos questionários. Para essas simulações, foram calculadas as percentagens de acertos do sistema fuzzy em relação às respostas dadas pelos especialistas. Abaixo são apresentados os resultados e as discussões de cada simulação.

TABELA 1. Valores de temperatura do ar e concentração de amônia medidos no interior da câmara climática e frequências e tempos médios normalizados, de expressão dos comportamentos observados. Values of air temperature and ammonia concentration measured indoor of the climatic chamber and normalized frequencies and average times, of the observed behaviors.

\begin{tabular}{cccccccc}
\hline Condição & $\begin{array}{c}\text { Temperatura } \\
\left({ }^{\circ} \mathrm{C}\right)\end{array}$ & $\begin{array}{c}\text { Concentração } \\
\text { de Amônia } \\
(\mathrm{ppm})\end{array}$ & Deitar & Ciscar & Ninho & $\begin{array}{c}\text { Limpar } \\
\text { Penas }\end{array}$ & Bebedouro \\
\hline 1 & 26,3 & 8 & 0,23 & 1,00 & 0,40 & 0,53 & 0,16 \\
2 & 26,3 & 21 & 0,33 & 0,24 & 1,00 & 0,16 & 0,00 \\
3 & 26,3 & 18 & 0,00 & 0,24 & 0,20 & 0,41 & 0,28 \\
4 & 29,5 & 18 & 0,00 & 0,52 & 0,00 & 0,72 & 0,28 \\
5 & 29,5 & 18 & 0,67 & 0,00 & 0,30 & 0,33 & 0,51 \\
6 & 29,5 & 26 & 0,23 & 0,00 & 0,00 & 0,20 & 0,47 \\
7 & 33 & 25 & 1,00 & 0,00 & 0,60 & 0,00 & 1,00 \\
8 & 33 & 6 & 0,67 & 0,00 & 0,10 & 0,27 & 0,76 \\
9 & 33 & 12 & 0,45 & 0,00 & 0,20 & 1,00 & 0,75 \\
\hline
\end{tabular}

Primeira simulação: Após a inserção das primeiras 25 regras descritas na primeira etapa, foi realizada simulação utilizando os dados experimentais. Nessa simulação, não se pode concluir nada a respeito do grau de explicação dos dados pelo sistema fuzzy, uma vez que os valores foram todos iguais a 0,5 . Portanto, nessa primeira simulação, o conjunto de regras foi considerado insuficiente.

Segunda simulação: A segunda simulação foi realizada com o sistema fuzzy possuindo 35 regras ( 25 da primeira etapa e dez da segunda). Comparando-se a saída do sistema com as respostas médias dos especialistas, obteve-se o resultado de 44,4\% de acerto. Esse resultado, comparado com 73\% de explicação obtida no sistema fuzzy de BROWN-BRANDL et al. (2005), está muito baixo. No entanto, quando as respostas de saída do sistema são comparadas apenas com as respostas do especialista número 2, o qual foi constatado que possui maior tempo de experiência no manejo dessa linhagem Hybro-PG, verifica-se que o grau de explicação do modelo aumenta para 55,6\%. Esse resultado ainda é considerado baixo, porém denota a importância da experiência do especialista para a construção das regras.

Terceira simulação: A terceira simulação realizada, na qual o sistema fuzzy possuía o total de 117 regras (sendo 35 regras da simulação anterior mais 82 regras construídas a partir das simulações equivocadas da terceira etapa), obteve-se explicação de 55,6\% dos dados. A mesma percentagem de acerto foi constatada quando se comparou com as respostas fornecidas pelo especialista número 2. Quando o resultado da terceira simulação é comparado com os resultados obtidos nos modelos de BROWN-BRANDL et al. (2005) e AERTS et al. (2003), verifica-se que o grau de explicação desse sistema ainda é baixo e que as 82 regras provenientes do cálculo equivocado das frequências de ciscar pouco contribuíram para o aumento da taxa de acertos do sistema. 
Quarta simulação: A quarta simulação o sistema fuzzy possuía o total de 87 regras (sendo 35 regras somadas às 52 regras construídas a partir de situações corretas simuladas na terceira etapa). Verificou-se que, para essa simulação, o índice de acerto da saída do sistema fuzzy foi igual a 66,7\% e, portanto, maior que o índice encontrado na terceira simulação. Esse resultado confirma a hipótese inicial de que a simulação de valores utilizando modelos estatísticos possibilita a geração de regras que explicam situações com maior chance de ocorrerem, pois mesmo o sistema possuindo número menor de regras, conseguiu melhorar a explicação dos dados cerca de dez pontos percentuais, comparado com a terceira simulação. Comparando com a literatura, esse sistema já se aproxima dos resultados obtidos por BROWN-BRANDL et al. (2005).

Quinta simulação: A quinta simulação foi realizada usando todas as regras construídas, ou seja, 169 regras. Nessa simulação, observou-se que a saída do sistema fuzzy aderiu 77,8\% com as respostas dadas pelos especialistas para as situações experimentais. Esse resultado iguala-se ao encontrado por BROWN-BRANDL et al. (2005) para a estimativa do bem-estar de bovinos. Na TABELA 2, são apresentados os resultados das cinco simulações.

TABELA 2. Resultados das simulações com os dados experimentais. Results of the simulations with the experimental data.

\begin{tabular}{|c|c|c|c|c|c|c|c|c|c|c|c|}
\hline \multirow{3}{*}{$\begin{array}{c}\text { Condição } \\
1\end{array}$} & \multirow{3}{*}{$\begin{array}{c}\begin{array}{c}\text { Bem-Estar } \\
\text { (opinião dos } \\
\text { especialistas) }\end{array} \\
\text { Bom }\end{array}$} & \multirow{2}{*}{\multicolumn{2}{|c|}{$\frac{1^{\text {a }} \text { Simulação }}{\text { SC25 }}$}} & \multirow{2}{*}{\multicolumn{2}{|c|}{$\frac{2^{\mathrm{a}} \text { Simulação }}{\text { SC35 }}$}} & \multirow{2}{*}{\multicolumn{2}{|c|}{$\frac{3^{3} \text { Simulação }}{\text { SC117 }}$}} & \multirow{2}{*}{\multicolumn{2}{|c|}{$\frac{4^{a} \text { Simulação }}{\text { SC87 }}$}} & \multirow{2}{*}{\multicolumn{2}{|c|}{$\begin{array}{c}5^{\text {a }} \text { Simulação } \\
\text { SC169 }\end{array}$}} \\
\hline & & & & & & & & & & & \\
\hline & & 0,50 & (Bom) & 0,66 & (Bom) & 0,56 & (Bom) & 0,66 & (Bom) & 0,56 & (Bom) \\
\hline 2 & Bom & 0,50 & (Bom) & 0,09 & $\begin{array}{l}\text { (Muito } \\
\text { Ruim) }\end{array}$ & 0,20 & (Ruim) & 0,09 & & 0,20 & (Ruim) \\
\hline 3 & Ruim & 0,50 & & 0,31 & (Ruim) & 0,33 & & 0,31 & & 0,33 & \\
\hline 4 & Ruim & 0,50 & (Bom) & 0,32 & (Ruim) & 0,32 & (Ruim) & 0,32 & (Ruim) & 0,32 & (Ruim) \\
\hline 5 & Ruim & 0,35 & (Ruim) & 0,32 & (Ruim) & 0,44 & (Ruim) & 0,32 & (Ruim) & 0,44 & (Ruim) \\
\hline 6 & Ruim & 0,50 & (Bom) & 0,10 & $\begin{array}{l}\text { (Muito } \\
\text { Ruim) }\end{array}$ & 0,25 & (Ruim) & 0,18 & $\begin{array}{l}\text { (Muito } \\
\text { Ruim) }\end{array}$ & 0,25 & (Ruim) \\
\hline 7 & Bom & 0,50 & (Bom) & 0,09 & $\begin{array}{l}\text { (Muito } \\
\text { Ruim) }\end{array}$ & 0,09 & (Ruim) & 0,09 & $\begin{array}{l}\text { (Muito } \\
\text { Ruim) }\end{array}$ & 0,09 & $\begin{array}{l}\text { (Muito } \\
\text { Ruim) }\end{array}$ \\
\hline 8 & Bom & 0,50 & (Bom) & 0,34 & (Ruim) & 0,34 & (Ruim) & 0,54 & (Bom) & 0,54 & (Bom) \\
\hline 9 & Bom & 0,50 & (Bom) & 0,32 & (Ruim) & 0,32 & (Ruim) & 0,47 & (Bom) & 0,47 & (Bom) \\
\hline
\end{tabular}

Células sombreadas de verde constituem os acertos do sistema fuzzy e células sombreadas de vermelho constituem os erros.

Validação do sistema fuzzy: No mesmo período em que ocorreram os registros dos dados experimentais, foi solicitado para um funcionário da granja cedente das matrizes pesadas, que observasse alguns comportamentos previamente indicados, no galpão de produção de origem das aves utilizadas no experimento. Portanto, essas matrizes pesadas observadas na granja pertencem ao mesmo lote das aves observadas no experimento.

Essas observações foram realizadas conforme metodologia descrita por PEREIRA (2005), ou seja, as observações foram realizadas por período contínuo de 15 minutos na parte da manhã e 15 minutos contínuos na parte da tarde, em grupo de matrizes de número conhecido. Esse funcionário fez os registros em três dias e anotou os valores de temperatura no momento de cada observação. Porém, assim como foi feito com os dados experimentais, foram aproveitados apenas os dados do período da tarde por não terem interferência do arraçoamento.

Devido à coleta desses dados terem ocorrido concomitantemente com o experimento, alguns comportamentos que foram observados na câmara climática, e que não haviam sido previstos anteriormente na ficha de observação elaborada para os funcionários das granjas, não tiveram as frequências e tempos médios de ocorrências registrados. Porém, o sistema fuzzy proposto pode ser utilizado mesmo quando não se tiver valores observados para todas as variáveis de entrada, proporcionando grande vantagem para o seu uso. 
Caracterizadas as limitações dos dados obtidos para a validação, na TABELA 3 apresentamse os valores anotados na granja e que foram utilizados para validar o sistema fuzzy, já normalizados para os intervalos de $[0,1]$ pelas equações de isomorfismo.

TABELA 3. Frequências e tempos médios normalizados dos comportamentos observados na granja. Frequencies and average times normalized of the behaviors observed in the farm.

\begin{tabular}{cccccccccc}
\hline Dia & $\begin{array}{c}\text { Temperatura } \\
\end{array}$ & Deitar & Ciscar & Ninho & $\begin{array}{c}\text { Limpar } \\
\text { Penas }\end{array}$ & Bebedouro & \multicolumn{3}{c}{ Simulação do Bem-Estar } \\
\cline { 7 - 10 } & 22 & 0,93 & 0,70 & - & 0,50 & - & 0,66 & (Bom) & Bom \\
2 & 24 & 1,00 & 1,00 & - & 0,88 & - & 0,66 & (Bom) & Bom \\
3 & 26 & 0,46 & 0,56 & - & 0,81 & - & 0,66 & (Bom) & Bom \\
\hline
\end{tabular}

Devido à indisponibilidade de equipamento para registrar a concentração de amônia no galpão, esta medida foi estimada como baixa para os três dias de registros, baseando-se nos relatos e nas observações feitas por MIRAGLIOTTA (2000). Estes valores foram simulados no sistema fuzzy com 169 regras, testado na quinta simulação, e os resultados obtidos tiveram aderência de $100 \%$ com as respostas dadas por um especialista. Apesar de serem poucas simulações, considerou-se que a aderência da estimativa fornecida pelo sistema com as expectativas relatadas pelo especialista indica a sua validação.

O comportamento dos animais é uma janela entre o organismo vivo e o ambiente (GRAVES, 1982), que é composto pelas variáveis climáticas e sociais. Portanto, o ambiente influencia fortemente no comportamento do animal. Os animais sofrem a atuação do ambiente de forma positiva ou negativa, e os reflexos dessa atuação são as diversas alterações em seus mecanismos (biológicos, morfológicos e/ou fisiológicos), expressos em seus comportamentos.

De acordo com YAHAV et al. (1995), a temperatura crítica alta para a produção de frangos de corte em situação de crescimento/terminação é de $29{ }^{\circ} \mathrm{C}$, e a umidade relativa, com valores acima de $70 \%$. PEREIRA \& NÄÄS (2008) afirmaram que, a partir dos $29^{\circ} \mathrm{C}$, matrizes pesadas saem da zona de termoneutralidade e entram para a área crítica, não descartando o fato de que diferentes linhagens possuem diferenças quanto aos limites de termoneutralidade.

A sensação de bem-estar é complexa, sendo resultado do efeito de diferentes variáveis ambientais e sociais sobre as aves. O controle da temperatura dentro de um limite de termoneutralidade contribui, mas não esgota a necessidade de ambiente às aves. Nesses casos, onde só há o controle da temperatura, a estimativa de bem-estar carrega alto grau de imprecisão. $\mathrm{O}$ sistema fuzzy proposto, por considerar os comportamentos expressos pelo grupo à estimativa do bem-estar, trata melhor os efeitos de outras variáveis, mesmo que indiretamente, o que lhe confere maior grau de precisão.

Os efeitos de outras variáveis no bem-estar podem ser vistos nos resultados das simulações. As condições 8 e 9, que foram obtidas com temperatura ambiente de $33{ }^{\circ} \mathrm{C}$, na opinião dos especialistas, o bem-estar das aves foi classificada como "Bom", mesmo com a temperatura fora da zona de termoneutralidade (PEREIRA \& NÄ̈̈S, 2008; YAHAV et al., 1995). O inverso é observado para as condições 4; 5 e 6 , em que o bem-estar foi classificado como "Ruim", mesmo com a temperatura do ar menor, próxima do limite superior da termoneutralidade. Analisando o mesmo conjunto de dados, observa-se que isso ocorre porque as condições 4; 5 e 6 combinam altas temperaturas (acima da zona de termoneutralidade) com maiores concentrações de amônia no ar.

Alguns trabalhos (WATHES et al., 2002; McKEEGAN et al., 2005) observaram os efeitos negativos de concentrações de amônia acima de 20 ppm nos comportamentos de poedeiras e, apesar de os especialistas não conhecerem o ambiente térmico e aéreo dos vídeos, esses identificaram, nos comportamentos expressos pelas matrizes pesadas, menor grau de bem-estar quando a concentração de amônia foi próxima ou acima de 20 ppm. 
O temperamento das aves varia entre linhagens. O estudo comparativo do comportamento entre linhagens é importante para que se entendam os efeitos do ambiente no animal. Alguns autores têm estudado comparativamente os comportamentos afetados pelo ambiente (KEER-KEER et al., 1996; McGARY et al., 2003), mostrando diferenças importantes nas respostas comportamentais entre as linhagens. O sistema fuzzy proposto foi desenvolvido para matrizes pesadas da linhagem Hybro-PG, com 29 semanas de idade e, devido às diferentes respostas comportamentais das aves de diferentes linhagens e de diferentes idades, esse sistema não pode ser imediatamente utilizado para qualquer grupo de matrizes pesadas. Porém, a metodologia empregada na construção desse sistema pode ser automatizada em programa de computador e reproduzida para outros grupos de matrizes pesadas, possibilitando construir para cada grupo de aves o seu próprio sistema fuzzy para estimativa do bem-estar.

\section{CONCLUSÕES}

A metodologia de construção do sistema de suporte a decisão com base na teoria dos conjuntos fuzzy para estimativa do bem-estar de matrizes pesadas da linhagem Hybro-PG foi eficiente e permite ser atualizado facilmente. O grau de explicação do sistema fuzzy relaciona-se diretamente com a quantidade de regras do mesmo. Atualmente, o sistema possui o total de 169 regras e demonstrou nos testes realizados, aderência de $77,8 \%$ com resultados preditos por especialistas.

Esse sistema fuzzy de estimativa do bem-estar de matrizes pesadas pode auxiliar a tomada de decisão no acionamento de sistemas de climatização em aviários, estimando o bem-estar de matrizes pesadas em função de situações comportamentais e ambientais observadas no galpão.

\section{AGRADECIMENTOS}

À granja São José e aos seus funcionários, pela valiosa contribuição para o desenvolvimento desta pesquisa, e ao programa PIBIC/CNPq da UNESP, pela bolsa de Iniciação Científica concedida.

\section{REFERÊNCIAS}

AERTS, J.M.; WATHES, C.M.; BERCKMANS, D. Dynamic data-based modelling of heat production and groeth of broiler chickens: development of an integrated management system. Biossystems Engineering, Harpenden, v.84, n.3, p. 257-66, 2003.

AMENDOLA, M.; CASTANHO, M.J.; NÄÄS, I.A.; SOUZA, A.L. Análise matemática de condições de conforto térmico para avicultura usando a teoria dos conjuntos fuzzy. Biomatemática, Campinas, v.14, p.87-92, 2004.

AMENDOLA, M.; SOUZA, A.L. Manual do uso da teoria dos conjuntos fuzzy no MATLAB 6.1. Campinas: Faculdade de Engenharia Agrícola, UNICAMP, 2004. 30 p.

BRACKE, M.B.M.; HULSEGGE, B.; KEELING, L.; BLOKHUIS, H.J. Decision support system with semantic model to assess the risk of tail biting in pigs. 1. Modeling. Applied Animal Behaviour Science, Linköping, v.87, n.1, p. 31-44, 2004.

BROWN-BRANDL, T.M.; JONES, D.D.; WOLDT, W.E. Evaluating modeling techniques for cattle heat stress pretiction. Biossystems Engineering, Harpenden, v.91, n.4, p.513-24, 2005.

CAMPOS, E.J. O comportamento das aves. Revista Brasileira de Ciência Avícola, Campinas, v.2, n.2, p.93-113, 2000.

COSTA, M.J.R.P. Princípios de etologia aplicados ao bem-estar das aves. In: CONFERÊNCIA APINCO DE CIÊNCIA E TECNOLOGIA AVÍCOLA, 20., 2003, Campinas. Anais... Campinas: APINCO, 2003. p.169-77. 
DUNCAN, I.J.H.; MENCH, J.A. Behaviour as an indicator of welfare in various systems. In: EUROPEAN SYMPOSIUM ON POULTRY WELFARE, 4., 1993, Potters Bar. Proceedings... Potters Bar: Universities Federation for Animal Welfare, 1993. p.69-80.

GRAVES, H.B. Behavioral responses of poultry (chickens) to management systems. In: SYMPOSIUM OF MANAGEMENT OF FOOD PRODUCING ANIMALS, 1982, West Lafayette. Proceedings... West Lafayette: Purdue University, 1982. v.2, p.122-38.

KEER-KEER, S.; HUGHES, B.O.; HOCKING, P.M.; JONES, R.B. Behavioural comparison of layer and broiler fowl: measuring fear responses. Applied Animal Behaviour Science, Linköping, v.49, n.4, p.321-33, 1996.

LANZILLOTTI, H.; LANZILLOTTI, R. Análise sensorial sob o enfoque da decisão fuzzy. Revista de Nutrição, Campinas, v.12, n.2, p.145-57, 1999.

MACARI, M.; FURLAN, R.L.; GONZALES, E. Fisiologia aviária aplicada a frangos de corte. Jaboticabal: FUNEP/UNESP, 1994. 296 p.

McGARY, S.; ESTEVEZ, I.; RUSSEK-COHEN, E. Reproductive and agressive behaviour in male broiler breeders with varying fertility levels. Applied Animal Behaviour Science, Linköping, v.82, n.1, p.29-44, 2003.

McKEEGAN, D.E.F.; SMITH, F.S.; DEMMERS, T.G.M.; WATHES, C.M.; JONES, R.B. Behavioral correlates of olfactory and trigeminal gaseous stimulation in chickens, Gallus domesticus. Physiology \& Behavior, Hare, v.84, p.761-8, march. 2005.

MIRAGLIOTTA, M.Y. Avaliação dos níveis de amônia em dois sistemas de produção de frangos de corte com ventilação e densidade diferenciadas. 2000. 222 f. Dissertação (Mestrado em Construções Rurais e Ambiência) - Faculdade de Engenharia Agrícola, Universidade Estadual de Campinas, Campinas, 2000.

PEREIRA, D.F. Avaliação do comportamento individual de matrizes pesadas (frango de corte) em função do ambiente e identificação da temperatura crítica máxima. 2003. 174 f. Dissertação (Mestrado em Construções Rurais e Ambiência) - Faculdade de Engenharia Agrícola, Universidade Estadual de Campinas, Campinas, 2003.

PEREIRA, D.F. Metodologia para estimativa de bem-estar de matrizes pesadas utilizando monitoramento digital e construção de modelos de simulação. 2005. $138 \mathrm{f}$. Tese (Doutorado em Construções Rurais e Ambiência) - Faculdade de Engenharia Agrícola, Universidade Estadual de Campinas, Campinas, 2005.

PEREIRA, D.F.; NÄÄS, I.A. Estimating the thermoneutral zone for broiler breeders using behavioral analysis. Computers and Electronics in Agriculture, Davis, v.62, n.1, p.2-7, 2008.

PEREIRA, D.F.; NÄÄS, I.A.; ROMANINI, C.E.B.; SALGADO, D.D.; PEREIRA, G.O.T. Indicadores de bem-estar baseados em reações comportamentais de matrizes pesadas. Engenharia Agrícola, Jaboticabal, v.25, n.2, p.308-14, 2005.

TEETER, R.G.; BELAY, T. Broiler water balance and thermobalance during thermoneutral and high ambient temperature exposure. Poultry Science, Savoy, v.72, n.1, p.116-24, 1993.

WATHES, C.M.; JONES, J.B.; KRISTENSEN, H.H.; JONES, E.K.M.; WEBSTER, A.J.F. Aversion of pigs and domestic fowl to atmospheric ammonia. Transactions of the ASAE, St. Joseph, v.45, n.5, p.1.605-10, 2002.

YAHAV, S.; GOLDFELD, S.; PLAVNIK, I.; HURWITZ, S. Physiological responses of chickens and turkeys to relative humidity during exposure to high ambient temperatures. Journal of Thermal Biology, Durham, v.20, p.245-53, 1995. 